\title{
Varicose Veins, Retinal Tears and Deep Vein Thrombosis, New Insights into the Causes from Adams Disease
}

\author{
James David Adams Jr. \\ Department of Pharmacology and Pharmaceutical Sciences, School of Pharmacy, University of Southern \\ California, Los Angeles, CA, USA \\ Email: jadams@usc.edu
}

Received 14 May 2015; accepted 7 July 2015; published 10 July 2015

Copyright (C) 2015 by author and Scientific Research Publishing Inc. This work is licensed under the Creative Commons Attribution International License (CC BY). http://creativecommons.org/licenses/by/4.0/

c) (i) Open Access

\begin{abstract}
Adams disease is the consequence of overly active transient receptor potential cation channels (TRP). This case report presents a patient suffering from multiple vascular problems and may provide new insights into the causes of venous problems. Endothelial and smooth muscle cells in veins contain TRP. These channels when activated excessively result in calcium accumulation, oxygen radical formation and apoptosis of endothelial and smooth muscle cells. The death of critical cells in vein valves, may lead to varicose veins. The loss of endothelial cells in venules may lead to retinal tears. Damage to vein walls may lead to a deep vein thrombosis. Transient receptor potential cation channels may be new drug targets of interest in the treatment or prevention of these conditions.
\end{abstract}

\section{Keywords}

Transient Receptor Potential Cation Channels, Deep Vein Thrombosis, Varicose Veins, Retinal Tears, Adams Disease

\section{Introduction}

The cause of varicose veins is not known. About $60 \%$ of adults in the US have varicose veins. Women make up $75 \%$ of the cases. Diagnosis depends on ultrasound techniques to visualize the veins and other techniques. Leg veins contain smooth muscle cells that contract to push blood toward the heart. Valves in leg veins open and close with the contractions to prevent blood from flowing back into the legs. Age, pregnancy, being female or obese, cause the veins and vein valves to not work as they should, allowing blood to collect in the lower leg 
veins causing them to distend, contort and become varicose. Treatments include various plant derived medicines, compression therapy, sclerotherapy, surgical ligation with stripping, microphlebectomy, and endovenous laser treatment. Many of these therapies involve destroying or removing the varicose veins. Plant medicines and nutritional supplements used in the treatment of varicose veins include ginkgo biloba, vitamin K, coenzyme Q10, omega-3 fatty acids and horse chestnut seed extract. Patients are also encouraged to exercise, get rid of excess weight, not sit with the legs crossed, not stand for long periods of time, elevate the legs, eat a high fiber low sodium diet and other lifestyle changes.

The cause of retinal tears is not known. In many cases the vitreous humor shrinks away from the retina, pulling at the retina causing it to tear. When a retinal tear occurs with bleeding into the eye, veins under the retina, in the choroid, may rupture and send blood into the posterior eye chamber. Rupture of the veins also leads to blood flow behind the retina that can cause the retina to detach. Aging, eye surgery, eye trauma and a history of retinal tears may be risk factors for retinal tears. Treatment can involve laser welding of bleeding veins in the retina.

The cause of deep vein thrombosis can be blood clotting disorders in some patients. In most patients, the cause is not known. These clots usually form in deep veins in the legs and are diagnosed with the duplex ultrasound, X-ray venography or magnetic resonance imaging. Long periods of not moving legs, injury, trauma, surgery, pregnancy, birth control pills, hormone replacement therapy, obesity, smoking, cancer, heart failure, inflammatory diseases, nephrotic syndrome, infection, HIV, intravenous drug use, a family history of deep vein thrombosis and aging may increase the risk of deep vein thrombosis. Treatment involves warfarin, heparin, dabigatran, apixaban, enoxaparin, rivaroxaban, catheter directed thrombolysis and other treatments. Anticoagulant therapy typically lasts several months and can be dangerous, potentially causing bleeding episodes.

TRP are found in the plasma membranes of many cells in the body. It may respond to heat, cold, mechanical and other stimulation. TRP are the main pain sensing and transmitting receptors in the body and are abundant in skin sensory afferent neurons. Typically, when these cation channels open, calcium enters into cells. Excessive opening of the channels can cause apoptosis [1]. Veins contain TRP in smooth muscle cells and endothelial cells.

\section{Case Presentation}

Adams disease is a disease caused by excessively active TRP [2]. The disease is characterized by pain in the skin, cracking and bleeding of the skin and other conditions. This report describes a Caucasian man who suffers from Adams disease that has caused varicose veins in the left leg, a retinal tear in the left eye and a deep vein thrombosis in the left lower leg. Varicose veins caused the left leg and ankle to swell. Treatment involved compression stockings. The retinal tear was characterized by blood release into the left eye. The ruptured vein in the retina was successfully treated with laser coagulation. The patient still has floaters in the left eye that decrease visual acuity more than 2 years later. The deep vein thrombosis was characterized by pain and additional swelling in the left leg. An ultrasound scan demonstrated the thrombosis in the popliteal vein behind the knee. Enoxaparin and warfarin therapy were initiated. The patient's coagulation factors were examined and found to be normal, including D-dimer. The patient has no coagulation problems that may have caused the thrombosis or other venous pathology. Examination of the heart by echocardiography with Doppler ultrasound and ECG found a normal, athletically enlarged heart with no arrhythmias, flow irregularities or other problems. The patient is generally in good health. He is $1.8 \mathrm{~m}$ tall, weighs $74.8 \mathrm{~kg}$, has normal blood pressure, blood glucose and blood cholesterol. He runs $13 \mathrm{~km}$ every day and has been a runner for more than 34 years. Treatment with warfarin continued for 3 months with no reappearance of thrombosis. At that point, warfarin was discontinued.

\section{Discussion}

Many different forms of TRP are found in venous endothelial cells and smooth muscle cells [3]. These channels are activated by thermal stimulation, mechanical stimulation and endogenous compounds such as NO, oxygen free radicals, diacylglycerol, sphingosine, phospholipids, oxidized lipids, arachidonic acid, arachidonic acid metabolites, and prostaglandins. In addition, N-oleoylethanolamide, is a TRPV1 agonist [4]. 1-Oleoyl-2-acetyl-snglycerol is an agonist for TRPC3 and TRPC5. A-Linolenic acid is an agonist for and inducer of TRPC1 and TRPC5 [5] [6]. Arachidonic acid and C2-ceramide are inducers of TRPC6. GM1 gangliosides induce TRPC5. The inflammatory adipokine, vascular endothelial growth factor, induces TRPC5 and TRPC6. The prostaglandin PGE2 increases the activity of TRPC5. Most of the deep vasculature is surrounded by perivascular fat that se- 
cretes adipokines and fat metabolites. These oxidized fats, especially the arachidonic acid metabolites, may cause long term activation of TRP leading to cell death through apoptosis. Perivascular fat deposits increase with aging.

Cytochrome P450 derived arachidonic acid metabolites, the epoxyeicosatrienoic acids, regulate venous tone and angiogenesis through activation of TRPC6, a canonical channel [7]. Endocannabinoids such as N-arachidonoyl dopamine regulate endothelial TRPV1, a vanilloid channel, and decrease inflammation in veins [8]. Angiotensin II is an inflammatory adipokine secreted by visceral fat and perivascular fat. Angiotensin II induces TRPC1 and TRPC3 activity in venous smooth muscle cells [9].

The TRPs found in venous endothelial cells and smooth muscle cells include: TRPC1-7 except for TRPC2, TRPV1-4, the melastatin channels TRPM2-8 except for TRPM5, the ankyrin channel TRPA1, the polycystin channel TRPP2 and the mucolipin channel TRPML1 [3]. These channels may be interesting targets for drugs designed to treat or prevent varicose veins, deep vein thrombosis and retinal tears. Prevention of these conditions may depend on lifestyle changes to minimize perivascular fat deposits. Exercise decreases the activity of TRPC channels and is protective against vascular disease [6].

This case report presents a patient suffering from Adams disease that developed several venous pathologies. These pathologies may have been caused by TRP induced damage to endothelial cells and smooth muscle cells. Adams disease may provide new insights into the causes of some types of venous pathology.

\section{Conclusions}

This report provides new insights into possible causes of venous pathology. TRP may be drug targets of interest in interventions for these conditions. In addition, lifestyle changes including exercise may be of use in decreasing the incidence of venous pathologies.

\section{References}

[1] Adams, J. (2013) Editorial: Apoptosis Is Critical to Pain Control. Open Journal of Apoptosis, 2, 23-24. http://dx.doi.org/10.4236/ojapo.2013.23003

[2] Adams, J. (2011) Adams Disease: Description and Management with Capsaicin and Heat. Open Complementary Medicine Journal, 3, 10-12. http://dx.doi.org/10.2174/1876391X01103010010

[3] Yue, Z.C., Xie, J., Ye, A.S., Stock, J., Du, J.Y. and Yue, L.X. (2015) Role of TRP Channels in the Cardiovascular System. American Journal of Physiology Heart Circulatory Physiology, 308, H157-H182. http://dx.doi.org/10.1152/ajpheart.00457.2014

[4] Liu, D.Y., Zhu, Z.M. and Tepel, M. (2008) The Role of Transient Receptor Potential Channels in Metabolic Syndrome. Hypertension Research, 31, 1989-1995. http://dx.doi.org/10.1291/hypres.31.1989

[5] Sukumar, P., Sedo, A., Li, J., Wilson, L., O’Regan, D., Lippiat, J., Porter, K., Kearney, M., Ainscough, J. and Beech, D. (2012) Constitutively Active TRPC Channels of Adipocytes Confer a Mechanism for Sensing Dietary Fatty Acids and Regulating Adiponectin. Circulation Research, 111, 191-200. http://dx.doi.org/10.1161/CIRCRESAHA.112.270751

[6] Beech, D. (2013) Characteristics of Transient Receptor Potential Canonical Calcium Permeable Channels and Their Relevance to Vascular Physiology and Disease. Circulation Journal, 77, 570-579. http://dx.doi.org/10.1253/circj.CJ-13-0154

[7] Ding, Y.D., Fromel, T., Popp, R., Falck, J., Schunck, W. and Fleming, I. (2014) The Biological Actions of 11,12Epoxyeicosatrienoic Acid in Endothelial Cells Are Specific to the R/S Enantiomer and Require G(s) Protein. Journal of Pharmacology and Experimental Therapeutics, 350, 14-21. http://dx.doi.org/10.1124/jpet.114.214254

[8] Wilhelmsen, K., Kkakpour, S., Tran, A., Sheehan, K., Schumacher, M., Xu, F.Y. and Hellman, J. (2014) The Endocannabinoid/Endovanilloid N-Arachidonoyl Dopamine (NADA) and Synthetic Cannabinoid WIN55,212-2 Abate the Inflammatory Activation of Human Endothelial Cells. Journal of Biological Chemistry, 289, 13079-13100. http://dx.doi.org/10.1074/jbc.M113.536953

[9] Zhu, Z.M., Luo, Z.D., Ma, S.T. and Liu, D.Y. (2011) TRP Channels and Their Implications in Metabolic Diseases. Pflugers Archives European Journal of Physiology, 461, 211-223. http://dx.doi.org/10.1007/s00424-010-0902-5 